\title{
Study of Microdispersion of Filler in Polymers by AFM Image Analysis
}

\author{
C. C. Wang*, J. B. Donnet**, S. H. Wu*, T. K. Wang*** \\ * University of Yantai, 264005 Yantai, P.R.China \\ ** University of Mulhouse, 3, rue A. Werner, 68093 Mulhouse Cedex, France \\ *** University of Le Havre, 25, rue Philipe Lebon, 76058 Le Havre, France
}

It is well known that the dispersion state can influence deeply several important properties in the application, such as abrasion, fuel consumption, and fatigue, etc. For these reasons filler dispersion has been studied since a long time [1-3]. In recent year, the atomic force microscopy (AFM) becomes a new tool for filler dispersion analysis [4-6]. In this work, we used the AFM technique for filler dispersion observation at microscopic level and the image analysis tool to get some quantitative information.

Five carbon blacks were used according to their grades to form two series for comparison: one is the carbon blacks with different primary particle sizes but similar structure (N115, N330 and N549), another is with similar primary particle size but different structure (N326, N330 and N347). The rubber samples were prepared in an internal blender. The blending was made under the conditions to have a good microdispersion without undispersed agglomerates. The samples were observed with Nanoscope IIIa (Digital Instruments, U.S.A) in Height and phase modes.

In the AFM phase images, the filler appears as clear spots on a dark background formed by the polymer matrix. In general, the clear spots don't represent the whole aggregates since they are partly burried in the matrix. Even for carbon blacks with different structures, it appears that the higher structure (N347>N330 $>$ N326) corresponds to finer particles and higher number density. This could be related to the AFM method that detects preferentially the extremities of the aggregates exposed to the sample surface.

Intuitively the surface fraction occupied by the particles $\left(F_{p}\right)$ is related to the filler loading. However, it is difficult to predict quantitatively the correlation which is influenced by several factors. A simple theoretical simulation can help us to treat the extreme cases. Suppose that in a bulk sample there are $n$ particles at a fixed layer thickness and they are distributed randomly. In case that the new surface preparation, if the filler particles can be cut in the same way as the polymer matrix, it can be deduced that the surface fraction is equal to the volume fraction. However, normally the carbon black particles are rarely broken during the surface preparation. The aggregates on the cutting line will be on one of the two new surfaces generated. In this case, the theoretical surface fraction in the images will be the half of the volume fraction.

Apart from the main contribution of filler loading on the surface fraction in the images, there are still several other factors which tend to enlarge the fraction. When the matrix polymer has a good affinity with the filler, it is possible that a layer of polymer is adhered. For the filler particles protruding from the matrix surface, it is known that the image is enlarged more or less because of the AFM tip geometry. Furthermore, it is shown that the AFM phase image can reveal the filler particles near the matrix surface. For the same reason the larger "immersed" part of a surface 
particle can also be detected. These factors contribute to a larger surface fraction of filler in the images than the theoretical prediction and it is hard to separate these effects.

At the microscopic level, the particules size distribution is an indicator of the microdispersion. Since the carbon blacks in our samples are well dispersed as a starting point for AFM image analysis, it is interesting to compare the effect of the carbon black grades. The particle size distributions follow well the carbon black grades (Fig.1). It appears that the observed particles present nearly the double of the primary particle size and a fraction of that of the aggregates. This indicates a good dispersion of fillers in the samples.

For different microdispersion states, it is posible to compare them by analyzing the AFM images. The comparison of bad dispersion case with one good dispersion case in this work (N330) is plotting the accumulated surface area of the filler as a function of the area of individual aggregate (agglomerate). The case of good dispersion is characterized by a fast level-off of the curve at small area, while the curve for the bad dispersion continue to increase till big area.

In conclutions, the AFM images can give a direct indication of the microdispersion state of filler in rubber and sufficient image number is necessary for obtaining the statistical values. The filler fraction in AFM images is well related to the loading in the recipe, but the quantitative correlation is influenced by several factors.

\section{References}

[1] A.Y. Coran and J.B. Donnet, Rubber Chemistry and Technology, 65 (1992) 998.

[2] M. Gerspacher and C.P. O'Farreli, Elastomerics, 4(1991) 35.

[3] W.M. Hess, Rubber Chemistry and Technology, 64(1991) 386.

[4] S. Maas and W. Gronski, Kautschuk Gummi Kunststoffe, 47 (1994) 409.

[5] A. Lapra, These, Universite of Pierre et Marie Curie, France, 1999.

[6] J. B. Donnet et al., Kautschuk Gummi Kunststoffe, 52 (1999) 340.

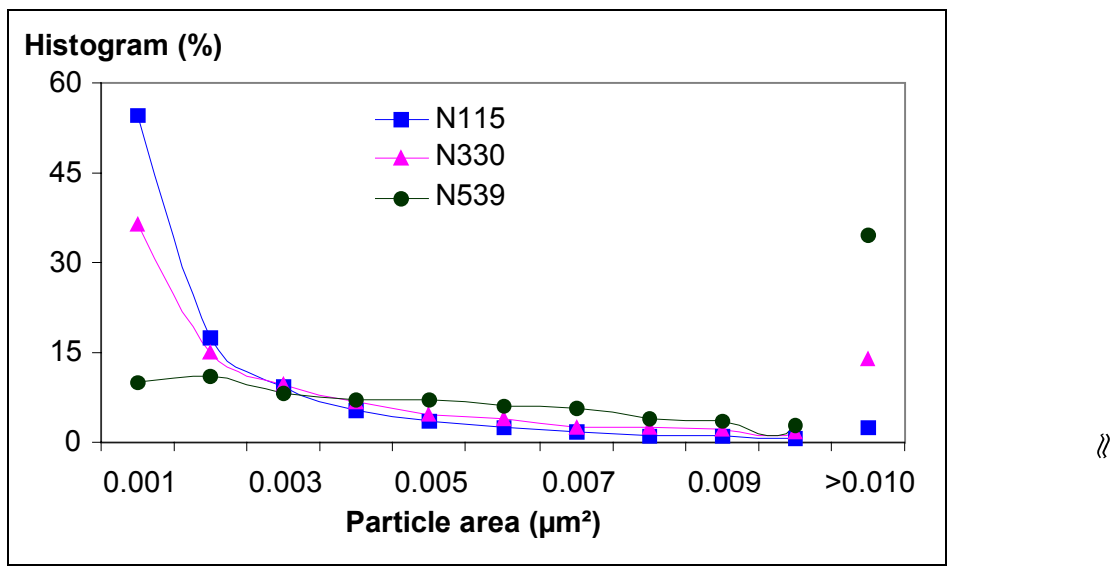

Fig.1 Particle size distribution of N115, N330 and N539 obtained by AFM image analysis. 